\title{
X1: A Robotic Exoskeleton for In-Space Countermeasures and Dynamometry
}

\author{
Rochelle Rea ${ }^{1}$, Christopher Beck ${ }^{1}$, and Roger Rovekamp ${ }^{1}$ \\ Oceaneering Space Systems, Houston, TX 77058 \\ Myron Diftler ${ }^{2}$ \\ NASA Johnson Space Center, Robotic Systems Technology Branch (ER4), Houston, TX 77058 \\ and \\ Peter Neuhaus ${ }^{3}$ \\ Florida Institute for Human and Machine Cognition, Pensacola, FL, 32502
}

\begin{abstract}
Bone density loss and muscle atrophy are among the National Aeronautics and Space Administration's (NASA) highest concerns for crew health in space. Countless hours are spent maintaining an exercise regimen aboard the International Space Station (ISS) to counteract the effect of zero-gravity. Looking toward the future, NASA researchers are developing new compact and innovative exercise technologies to maintain crew health as missions increase in length and take humans further out into the solar system. The X1 Exoskeleton, initially designed for assisted mobility on Earth, was quickly theorized to have far-reaching potential as both an in-space countermeasures device and a dynamometry device to measure muscle strength. This lower-extremity device has the ability to assist or resist human movement through the use of actuators positioned at the hips and knees. Multiple points of adjustment allow for a wide range of users, all the while maintaining correct joint alignment. This paper discusses how the X1 Exoskeleton may fit NASA's onorbit countermeasures needs.
\end{abstract}

\section{Introduction}

A s humans continue to push the boundaries of space travel, ensuring the health of the crew will continue to be one of the foremost challenges that physicians and scientists must undertake. As we explore beyond low earth orbit, the effects of reduced gravitational force over longer durations of space travel will contribute to even greater muscle strength degradation and increased bone density loss. This phenomenon is well known and documented. ${ }^{1-3}$ As a result, much effort has been exerted to maintain crew health using a series of exercise, or countermeasure, devices currently aboard the ISS such as the Advanced Resistive Exercise Device (aRED), the Combined Operational Load-Bearing External Resistance Treadmill (COLBERT), and the Stationary Bike. Through daily exercise regiments, these devices were designed to counter the effects of microgravity on bone density and muscle loss and to ensure shorter rehabilitation times upon return to Earth. However, their relatively large size and limited data collection capability do not make them the best choice for future long range exploration missions.

$\mathrm{X} 1$ is a new robotic exoskeleton that not only provides mobility for humans on earth, but is also being evaluated as an in-space countermeasures and dynamometry device. Lower-extremity exoskeletons are quickly becoming a staple area of research, rehabilitation, and mobility assistance across the world. The multitude of different devices currently available for these applications include the HULC, Ekso, Sarcos Exoskeleton, BLEEX, HAL 5, and the ReWalk to name a few. Each of these exoskeletons has their place in the field of mobility devices, all with their own niche. Some are used as load-carrying devices, such as the HULC ${ }^{4}$, while others specialize in rehabilitation and paraplegic assistance such as Ekso ${ }^{5}$ and $\mathrm{ReWalk}^{6}$. The X1, initially designed as a mobility device for persons with paraplegia, is now being tailored to address the needs of on-orbit countermeasures and crew health maintenance.

\footnotetext{
${ }^{1}$ Robotics Engineer, Robotics Systems and Technology Branch, ER4

${ }^{2}$ Robonaut and X1 Exoskeleton Project Manager, Robotics Systems and Technology Branch, ER4

${ }^{3}$ Research Scientist
} 
Using X1 to provide muscle resistance at the knees, hips, and possibly ankles could allow for common crew exercises, both concentric and eccentric, to be performed with a small and portable device. Additionally, X1 provides significantly increased real-time feedback to close the exercise prescription loop with physicians on the ground. An easily adaptable system, the X1 allows for resistances (torque) and automated movements to be adjusted in real-time, and also allows for the application of isokinetic, isotonic, and isometric muscle strength assessments.

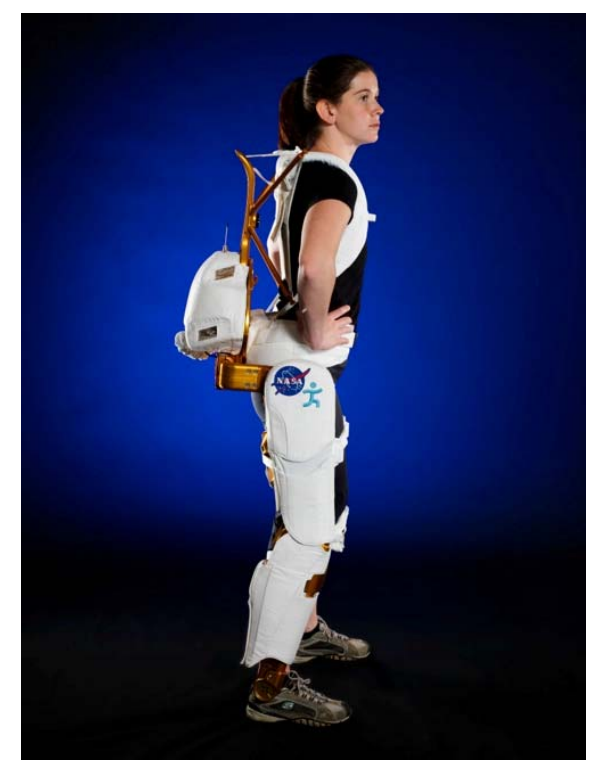

Figure 1: NASA’s X1 Exoskeleton built in partnership with IHMC, 2012

\section{X1 Background}

The X1 Exoskeleton was developed in the Dexterous Robotics Lab (DRL) at the NASA Johnson Space Center in Houston, TX in partnership with the Florida Institute for Human and Machine Cognition (IHMC) in Pensacola, FL. $\mathrm{X} 1$ is a technology spinoff from the Robonaut 2 (R2) Project, a humanoid robot currently operating aboard the International Space Station. ${ }^{7}$ Much of the X1 base architecture was leveraged from R2, including safety systems, basic actuator design, communication scheme, and embedded motor drive controls, which have all been tested and vetted on the ground and aboard the ISS.

In addition to technology derived from R2, work was also leveraged from the IHMC Mobility Assist Exoskeleton, which uses a similar series elastic actuation scheme. This exoskeleton was designed for applications here on Earth such as zero assistance control, gait rehabilitation, and assisted walking. ${ }^{8}$

The X1 exoskeleton currently has four active degrees of freedom (DOF) at the hips and the knees, with powered movement constrained to the sagittal plane. It also has six passive degrees of freedom for abduction and adduction; internal and external rotation; and dorsiflexion and plantarflexion. Any of these passive DOFs may be left free to move or locked out to intentionally constrain movement. Future improvements currently in work include exchanging the passive ankle for a powered ankle to allow for additional dynamometry evaluations and countermeasure exercises.

Worn around the legs with a set of cuffs, and around the torso with a backpack and hip belt, the tethered X1 weighs 57 pounds. It also has multiple adjustment points at the thighs, shins, and hips to accommodate a range of user spanning from the $5^{\text {th }}$ to $95^{\text {th }}$ percentile.

Each active joint is driven with a series elastic rotary actuator and custom designed embedded motor controller. Series elastic actuators are an ideal choice for actuation because they offer high fidelity impedance control. ${ }^{8}$ Each actuator may be controlled in a number of different states, such as impedance, position, and torque control and additional nested loops allow for safety and control limits including torque, velocity, and current. These parameters, as well as position and acceleration limits, can also be set in the high level controller to further ensure the safety of the user wearing the device. Other safety considerations include motion-stop controllers and integrated hardstops at each joint to prevent hyperextension or hyperflexion of the knees or hips. This combination of actuator and software design allow the $\mathrm{X} 1$ to safely achieve the significant torque loads at each joint that are necessary for countermeasures and dynamometry. 
Using custom controllers, the $\mathrm{X} 1$ has the ability to assist or resist a person's movements by simply altering parameters at the high level interface. This interface allows the user to control position, torque (resistance), or velocity. Providing control flexibility also allows for custom designed exercise routines to be written by countermeasure specialists for use in space, and custom designed rehabilitation routines to be written by therapists on the ground.

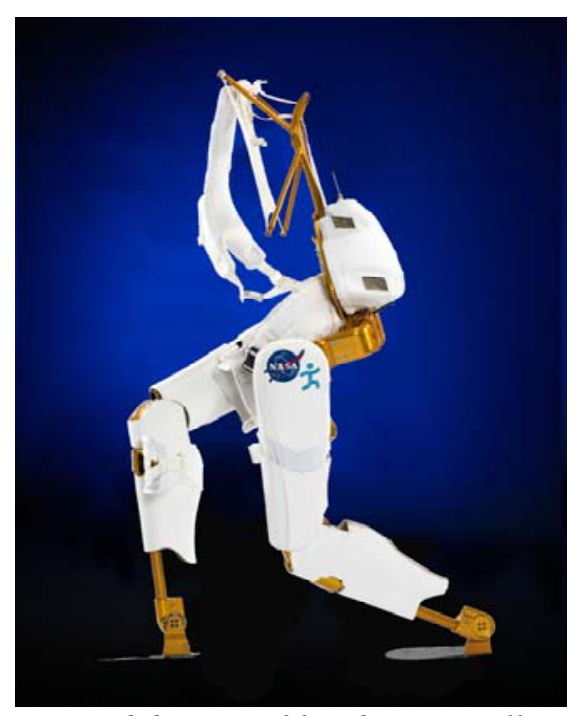

Figure 2: X1 photograph showing four actuated degrees of freedom, as well as the backpack which houses the high level computer, power distribution system, and safety system.

Because the X1 was designed to be lightweight, compact, and portable, it stands out as an attractive piece of hardware for spaceflight where volume and mass restrictions are of concern. Moreover, preliminary assessments have shown that the X1 can provide safe, efficacious exercise and dynamometry that could help astronauts maintain health during long-duration missions and afterward.

\section{On-Orbit Countermeasures}

Even on Earth, regular exercise is required to maintain a healthy lifestyle. However, we are fortunate that gravity continually resists our movements thus keeping our rate of bone loss equal to the rate of bone creation, even without a dedicated exercise regimen. On Earth "new bone is normally deposited in proportion to the compressional loads directed on a bone." ${ }^{\prime \prime}$ In space bone formation decreases rapidly because there are typically no constant compressional loads applied to the human body.

In addition to bone density loss, muscle atrophy is also of great concern during spaceflight. Like bone density loss, the effects of muscle atrophy have been widely documented. Fitts, et al. have shown, based on data from Skylab and Mir, muscle decline of up to 30\% during extended flight durations, and that this muscle atrophy leads directly to reduced peak force of a limb. ${ }^{10}$ In addition it has been documented that the loss of limb muscle mass is "exponential with the duration of flight."11 Based on this data and other documented cases and studies, it is understandable that physicians and physiologists are highly concerned with combating the effects of spaceflight on the human body. Even now, as we send our astronauts into space for up to six months, we can predict the effects that space travel will have as we explore beyond low earth orbit to asteroids, the moon, Mars, and beyond. 


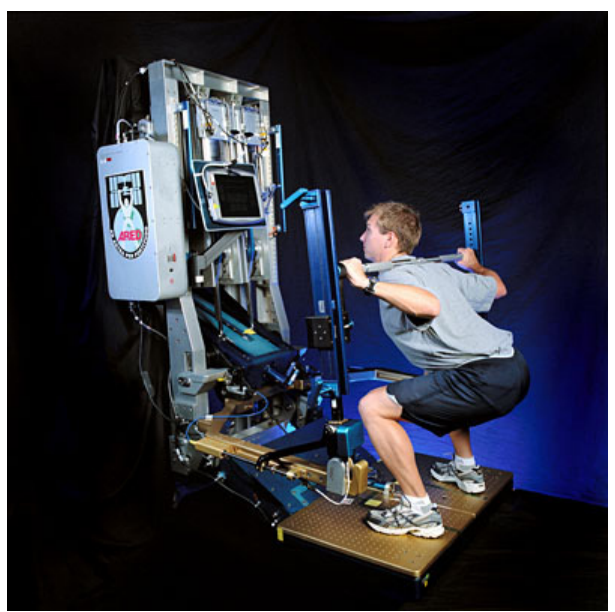

Figure 3: NASA's Advanced Resistive Exercise Device (ARED)

There are currently several existing countermeasure, or exercise, devices aboard the ISS. The most used device is the Advanced Resistive Exercise Device (ARED). ARED was designed at NASA-Johnson Space Center (JSC) to mitigate the loss of muscle mass and muscular strength associated with microgravity, while providing the ability to impart greater bone loading than its predecessor, the interim Resistive Exercise Device (iRED). The ARED device is designed to provide loads ranging from 0 to 600 pounds. A recent study examined the effects of spaceflight coupled with exercise using the ARED and iRED devices. It showed that astronauts using ARED for their exercise regiments returned to Earth with leaner muscles and "the mitigation of bone loss on the typical long-duration spaceflight missions of $\sim 180$ days could be controlled without the use of pharmaceutical agents."12 Although ARED has shown promising results as a countermeasure device, it has a great size and mass drawback. It is volumetrically large and requires a large vibration isolation system to minimize vibrations imparted into the ISS structure during workouts. It also restricts crew to a specific location within the station. In addition, its limited data feedback does not allow it to provide specific joint data or muscle strength assessments to the user or the physicians. It has been noted that physicians monitoring astronaut health on the ground would welcome such ability.

\section{X1 as a Countermeasures System}

Evaluations are ongoing at the Johnson Space Center to evaluate the X1 Exoskeleton as a countermeasure device for on-orbit exercise. As a lower extremity exoskeleton, X1 has the ability to impart desired torques at hip and knee joints, and its ability to be freely programmed allows for a myriad of exercises. Such resistance training could include hamstring curls and leg extensions, as well as coordinated exercises like straight-leg deadlifts.

For leg extension exercises, the X1 Exoskeleton could be operated in the following manner. After donning the suit, the user could set a specific "start" point for the extension. The user would then dial in a desired resistance, just as if they were at the local gym. This resistance would be applied until reaching a desired "stop” point at which point it could sense the user's intent to return to the starting position and decrease the resistance for the return travel. However, because the X1 Exoskeleton is so easily programmed, the user could desire to keep the desired resistance through the full motion of the exercise. In addition exercise profiles can be easily stored and recalled for later use or easily modified to create a suite of readily available regiments.

In addition to common crew exercises such as the ones mentioned above, the X1 Exoskeleton could fill the spaceflight void when it comes to eccentric exercises. Due to complete control at each joint, the X1 has the ability to perform both concentric and eccentric exercises with ease. Given a desired torque, a resistance could be applied to a joint during the concentric phase of an exercise, and a higher resistance could be applied during the eccentric phase; this type of exercise is classified as eccentric overload and is highly desired aboard ISS.

The authors are working closely with scientists from the Human Health and Performance group at NASA-JSC, in order to tailor the design of the X1, and its future iterations, to meet the specific needs of the crew. Recently X1 engineers have been evaluating the ability to accurately perform specific eccentric overload exercises like the one mentioned above. These types of exercise apply a greater force to muscles during the eccentric phase of motion and contribute to greater muscle development than standard concentric-only exercises. ${ }^{13}$ Although these types of exercise are currently a challenge for conventional exercise machinery on ISS, the versatility of the X1 system allows these unique exercise profiles to be programmed with relative ease. 


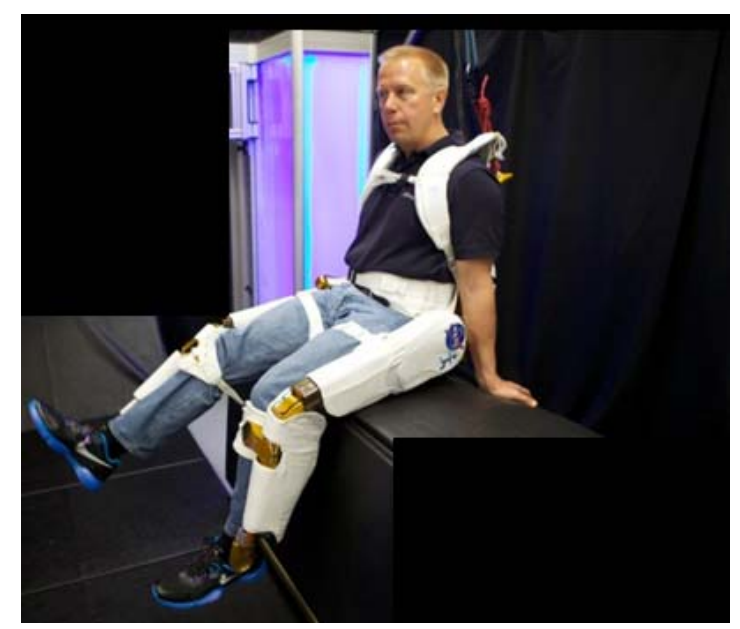

Figure 4: Astronaut Tim Kopra evaluating X1's leg extension exercise capability.

Currently the X1 has the ability to achieve over $300 \mathrm{Nm}$ of peak torque at each joint, and designs are in work to increase this capability to meet and surpass desired on-orbit exercise and dynamometry numbers. Beyond single joint exercises such as hamstring curls or leg extensions, X1 has the ability to perform coordinated joint exercises such as deadlifts, and future revisions may include other more complicated coordinated movements as deemed appropriate. With the high output torques at each actuator, X1 would be able to adequately exercise even the strongest users.

During each movement of an exoskeleton joint, whether for exercise or assisted mobility, joint data is streamed back to the brainstem computer in real-time. This data includes parameters such as position, velocity, acceleration, torque, rate of change of torque, etc., making it a valuable feedback tool that other on-orbit exercise devices lack. Using this data, physicians and exercise physiologists on the ground can evaluate how well both X1 and the user are performing the desired exercise, so exercise prescription changes can quickly be made if necessary.

As previously mentioned, a standout feature of the X1 is its small size and low profile. Low weight leads to low upmass and cost; its small profile could make it very appealing for longer distance space travel, where volume constraints inside a capsule would be even tighter. Because all the forces imparted in the X1 device are done so in a closed loop system, vibrations imparted into the vehicle are significantly reduced if not completely eliminated. It is possible that the wearer could be just lightly tethered to the vehicle. X1's compact profile also gives it the ability to be worn by the user while they are going about their normal everyday routines. Whether powered while doing a specific exercise, or unpowered and imparting resistance simply due to the back EMF of its motors, the X1 could allow for continual resistance to an astronaut while he or she is performing other mission-related tasks aboard the vehicle.

\section{Dynamometry}

As NASA continually strives to maintain crew health, physicians on the ground are also interested in health assessments of the crew before, during, and after spaceflight. Typical assessments done include isometric, isokinetic, and endurance assessments to evaluate muscle health and atrophy sustained during spaceflight. ${ }^{14,15}$ From these measurements, physiologists can extract data such as peak torque, angle of peak torque, time to peak torque, work, etc.

Currently aboard the ISS is the Muscle Atrophy Research and Exercise System (MARES) ${ }^{16}$, developed by the European Space Agency (ESA). It was delivered to ISS in 2010, for the potential applications of exercise and muscle strength evaluation. While it has shown promise for evaluating muscle strength, the device has garnered limited use due to evaluation times and performance concerns as compared to ground-based isokinetic dynamometers. Further, its large geometric volume make it problematic for long duration space missions.

Because the X1 Exoskeleton has the ability to collect joint data in real-time, physiologists on the ground can use this device to evaluate isometric, isokinetic, and other assessments of the crew while on-orbit and compare it to data measured on the ground during pre-flight assessments. Having the capability to continually monitor muscle strength will allow physicians and specialists on the ground to close the loop around exercise prescriptions and to adjust daily exercise regimens based on this feedback. 
In January of 2013, the X1 Exoskeleton as a knee dynamometer was compared to that of the Biodex system when used in the same manner. Eight subjects were studied on both devices for isokinetic and isometric knee flexion and extension. It was shown that the test-retest repeatability of peak torque of the X1 Exoskeleton was comparable to Biodex and other dynamometers previously tested at NASA. Given these test results, the Human Health and Performance division was satisfied with the results in comparison to Biodex testing, and it has given confidence that the X1 can meet in-flight dynamometry needs.

\section{Other Applications}

While the current X1 Exoskeleton is being tailored for in-space countermeasures and dynamometry, its potential applications on Earth are vast. For example, the $\mathrm{X} 1$ has the ability to act as a mobility assistance device for persons with lower extremity paralysis. Work is ongoing at IHMC to evaluate the device for this application and the results thus far have been promising. The ability of X1 to perform a sit-to-stand maneuver and allow a person with paraplegia to ascend a staircase is currently being investigated.

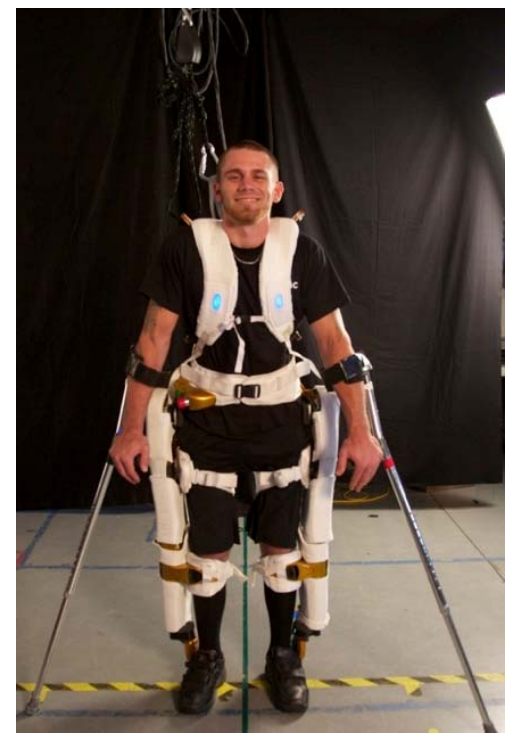

Figure 5: Mark Daniel using the X1 Exoskeleton to stand and walk

In space, this same assisted mobility could be used to augment human strength on missions to the moon and mars. Regardless of how much exercise the crew performs during a long-duration mission, they will likely welcome some assistance and/or strength augmentation when arriving at their destination. Additionally, the integration of X1 into future spacesuits is being considered. While NASA's spacesuit designs have come an impressively long way from their 1960's predecessors, astronauts may benefit from the added assistance while wearing the suit to more easily perform mundane extra-vehicular activity (EVA) tasks or to augment strength while carrying heavy loads.

In addition to assisted mobility and space applications, the $\mathrm{X} 1$ can potentially serve as a gait rehabilitation device for those suffering from paraparesis or hemiparesis due to stroke, spinal cord injury or other trauma. Given the vast programming ability of the X1 Exoskeleton, therapists could choose to program a resistance profile into each actuated joint based on user ability, and then adjust this profile as muscle strength improves. Because the X1 has already been designed with in-space exercise in mind, its joints have more than enough ability to provide great resistance on the ground for rehabilitation and exercise applications. Moreover, the accuracy of torque measurements demonstrated on X1 could provide the joint responsiveness necessary to accommodate those with very little ability to impart much force on their own.

\section{Ongoing Work}

Future improvements to the X1 Exoskeleton will increase its ability to act as both a countermeasures device as well as a dynamometer. Engineers are currently evaluating methods to add additional joints to X1 such as an ankle. Atrophy of the plantar flexors muscles (soleus, medial and lateral gastrocnemius) during long duration spaceflight remains a significant concern for crewmember health. ${ }^{17,18}$ By actively powering plantarflexion and dorsiflexion, users will be able to perform heel raise exercises at various joint angles to help maintain size and strength in the calf 
muscles. In fact, the gastrocnemius and soleus muscles have been historically hard to exercise in space and are prone to deconditioning during microgravity exposure. ${ }^{17}$

Studies began in June 2013 using MRI techniques to preliminarily evaluate the effects of the X1 ankle on the soleus and medial gastrocnemius muscle. This preliminary data suggests significant soleus and medial gastrocnemius muscle activation during bent leg X1 heel raises. Increases in the soleus muscle size post-exercise suggests enhanced metabolism and blood flow to the working muscle, which is essential for countermeasure applications.

Future studies will continue to evaluate the X1 Ankle for countermeasure and dynamometry applications. Dynamometry evaluations will be done by comparing the X1 performance to that of the Biodex machine; the current standard for measuring muscle strength at NASA. In addition, training studies and MRI techniques will also be used to evaluate calf muscle activation and strengthening over time.

In addition to ankle work, one of the most difficult aspects to designing an exoskeleton is to understand and implement the human-to-machine interface. This interface must be designed in such a way to correctly and efficiently impart forces from the exoskeleton into the human body. It must be comfortable for the user to wear for extended periods of time, yet be rigid enough to correctly maintain joint alignment. Designs are in work to create a more comfortable and conformal user interface for X1 specifically designed for on-orbit exercise.

\section{Conclusion}

As exoskeleton designs continue to evolve, it has been shown that their applications are not limited to useful work here on Earth. While most exoskeletons are designed for assisted mobility or strength augmentation, the NASA/IHMC X1 Exoskeleton is actively being designed for in-space exercise, countermeasures, and dynamometry. Creating a device that could one day be used for in-space countermeasures on long duration spaceflight, while at the same time allowing the ability to assist the astronaut when needed, will allow us to explore farther while still maintaining bone density, muscle strength, and overall astronaut health.

The notion of astronauts wearing exoskeletons to assist them in their daily work, provide them exercise, gather health data, aid their EVAs, and augment their strength on other planetary bodies, may still seem like science fiction to some. The authors believe, however, that these devices will soon become ubiquitous in the realm of spaceflight. Such a lightweight, low-profile, and versatile device will improve the lives of astronauts, allowing them to conduct better research and better exploration, which will directly improve the lives of all mankind.

The authors are confident that X1 will continue to reveal even more potential as an on-orbit exercise device. With the help of countermeasure specialists at NASA, continued evaluation and design improvements will make X1 not only the world's most versatile exoskeleton, but one of the spaceflight community's most capable device for exercise, health assessment, and astronaut assistance.

\section{Acknowledgments}

The authors of this paper would like to thank the Biomedical Research and Environmental Sciences Division of the Human Health and Performance Directorate at NASA-Johnson Space Center for their continued support of the $\mathrm{X} 1$ Exoskeleton and its prospective use as an in-space countermeasure and dynamometry device. Specifically the authors would like to acknowledge the hard work of Dr. John Dewitt, Dr. Kyle Hackney, Linda Loerch, Nathaniel Newby, and Dr. Lori Ploutz-Snyder, without whom ongoing testing and evaluation would not be possible. The X1 exoskeleton project was co-funded by NASA’s Office of Game Changing Development and the International Space Station Program Office.

\section{References}

\footnotetext{
${ }^{1}$ Caiozzo, V. J., Baker, M. J., Herrick, R. E., Tao, M., and Baldwin, K. M., "Effect of Spaceflight on Skeletal Muscle: Mechanical Properties and Myosin Isoform Content of a Sow Muscle,” Journal of Applied Physiology, Vol. 76, No. 4, April 1994, pp. 1764-1773.

${ }^{2}$ Morey, E. R., “Spaceflight and Bone Turnover: Correlation with a New Rat Model of Weightlessness,” BioScience Vol 29, March 1979, pp. 168-172.

${ }^{3}$ Morey, E. R., and Globus, R. K., "Hindlimb Unloading of Growing Rats: A Model for Predicting Skeletal Changes During Space Flight.,” Bone, Vol. 22, May 1998, pp. 83S-88S.

4“HULC,” URL: http://www.lockheedmartin.com/us/products/hulc.html [cited June 2013]

5“Exo Bionics For the Human Endeavor,” URL: http://www.eksobionics.com [cited June 2013]
} 
6“Bionics Research,” URL: http://www.rewalk.us [cited June 2013]

${ }^{7}$ Diftler, M.A, Mehling, J.S., Abdallah, M. E., Radford, N.A., Bridgewater, L.B, et al., "Robonaut2 - The First Humanoid Robot in Space,” IEEE International Conference on Robotics and Automation, Shanghai, China, May 2011.

${ }^{8}$ Kwa, H. K., Noorden, J. H., Missel, M., Craig, T., Pratt, J. E., and Neuhaus, P. D., "Development of the IHMC Mobility Assist Exoskeleton,” IEEE International Conference on Robotics and Automation, Kobe, Japan, 2009.

${ }^{9}$ Henderson, G. C., and Ueda, J.. "Pneumatically-Powered Robotic Exoskeleton to Exercise Specific Lower Extremity Muscle Groups in Humans" Fourth IEEE RAS/EMBS International Conference on Biomedial Robotics and Biomechatronics, Rome, Italy, June 2012.

${ }^{10}$ Fitts, R. H., Riley, D. R., and Widrick, J. J., "Functional and Structural Adaptations of Skeletal Muscle to Microgravity," Journal of Experimental Biology, Vol. 204, No. 18, 2001, pp. 3201-3208.

${ }^{11}$ Fitts, R. H., Riley, D. R., and Widrick, J. J., “Microgravity and Skeletal Muscle,” Journal of Applied Physiology, Vol. 89, 2000, pp. 823-839.

${ }^{12}$ Smith, S. M., Heer, M. A., Shackelford, L. C., Sibonga, J. D., Ploutz-Snyder, L., and Zwart, S. R., "Benefits for Bone From Resistance Exercise and Nutrition in Long-Duration Spaceflight: Evidence From Biochemistry and Densitometry,” Journal of Bone and Mineral Research, Vol. 27, August 2012.

${ }^{13}$ Friedmann, et al, "Effects of Strength Training With Eccentric Overload on Muscle Adaptation in Male Athletes."

European Journal of Applied Physiology, Vol. 108, March 2010, pp. 821-836.

${ }^{14}$ Laughlin, M. S., Lee, S. M. C., Loehr, J. A., and Amonette, W. E., "Isokinetic Strength and Endurance Tests Used Pre- and Post-Spaceflight: Test-Retest Reliability,” NASA TM-2009-214787, 2009.

${ }^{15}$ Gopalakrishnan, R., et al., "Muscle Volume, Strength, Endurance, and Exercise Loads During 6-Month Missions in Space,” Aviation, Space, and Environmental Medicine, Vol. 81, No. 2, February 2010.

16“"Muscle Atrophy Research and Exercise System (MARES),” URL:

http://www.nasa.gov/mission_pages/station/research/experiments/352.html [cited July 2013]

${ }^{17}$ Trappe, S., Costill, D., Gallagher, P., Creer, A., Peters, J. R., Evans, H., Riley, D. A., and Fitts, R. H., "Exercise in Space: Human Skeletal Muscle After 6 Months Aboard the International Space Station,” Journal of Applied Physiology, Vol. 106, April 2009, pp. 1159-1168.

${ }^{18}$ Fitts, R. H., Trappe, S. W., Costill, D. L., Gallagher, P. M., Creer, A. C., Colloton, P. A., Peters, J. R., Romatowski, J. G., Bain, J. L, and Riley, D. A. (2010) "Prolonged Space Flight-Induced Alterations in the Structure and Function of Human Skeletal Muscle Fibres,” Journal of Physiology, Vol. 588, Pt. 18, September 2010, pp. 3567-3592. 